\title{
Impact of Locus of Care on Outcomes in Adolescents and Young Adults with Osteogenic sarcoma and Ewing Sarcoma treated at Pediatric versus Adult Cancer Centers: An IMPACT Cohort Study
}

\author{
Mohammadreza Mortazavi ${ }^{1}$, Nancy Baxter ${ }^{2}$, Sumit Gupta ${ }^{1}$, Abha Gupta ${ }^{1}$, Cindy Lau ${ }^{3}$, \\ Chenthila Nagamuthu ${ }^{3}$, and Paul Nathan ${ }^{1}$ \\ ${ }^{1}$ The Hospital for Sick Children \\ ${ }^{2}$ The University of Melbourne School of Population and Global Health \\ ${ }^{3}$ Institute for Clinical Evaluative Sciences
}

September 25, 2021

\begin{abstract}
Abstract Background: Location of cancer care (LOC: pediatric versus adult center) impacts outcomes in adolescents and young adults (AYA) with some cancer types. Data on impact of LOC on survival in AYA with osteogenic sarcoma (OGS) and Ewing sarcoma (EWS) are limited. Objectives: To compare differences in demographics, disease/treatment characteristics, and survival in a population-based cohort of AYA with OGS or EWS treated at pediatric versus adult centers Methods: The IMPACT Cohort captured demographic, disease, and treatment data for all AYA (15-21 years old) diagnosed with OGS and EWS in Ontario, Canada between 1992-2012. Patients were linked to provincial administrative healthcare databases. Outcomes were compared between patients treated in pediatric versus adult centers using appropriate statistical methods. Results: 137 AYA were diagnosed with OGS (LOC: 47 pediatric, 90 adult) and 84 with EWS (LOC: 38 pediatric, 46 adult). AYA treated at pediatric centers were more likely to be enrolled in a clinical trial (OGS 55\% vs 1\%, [p $<0.001$ ]; EWS $53 \%$ vs $2 \%$, [p $<0.001$ ]) and received higher cumulative chemotherapy doses. Five-year event-free survival (EFS \pm Standard Error) in OGS and EWS were $47 \% \pm 4$ and $43 \% \pm 5$, respectively. In multivariable analysis, the impact of LOC (pediatric vs adult center) on EFS in OGS (adjusted hazard ratio [HR] 1.15, 95\% CI 0.58-2.27, P=0.69) and EWS (adjusted HR 1.82, 95\% CI 0.97-3.43, P=0.06) were not statistically significant. Conclusion: Outcomes did not differ by LOC suggesting that AYA with bone tumors can be treated at either pediatric or adult centers.
\end{abstract}

Oncology: Original research article

Impact of Locus of Care on Outcomes in Adolescents and Young Adults with Osteogenic sarcoma and Ewing Sarcoma treated at Pediatric versus Adult Cancer Centers: An IMPACT Cohort Study

Mohammadreza Mortazavi ${ }^{1,3} \mid$ Nancy N. Baxter ${ }^{3,4,7,8} \mid$ Sumit Gupta $^{1,2,3,4}$ | Abha A. Gupta ${ }^{1,5}$ | Cindy Lau $^{3}$ | Chenthila Nagamuthu ${ }^{3}$ | Paul C. Nathan ${ }^{1,2,3,4}$

${ }^{1}$ Division of Hematology/Oncology, The Hospital for Sick Children, Toronto, Ontario, Canada; ${ }^{2}$ Faculty of Medicine, University of Toronto, Toronto, Ontario, Canada $;^{3}$ Cancer Research Program, Institute for Clinical Evaluative Sciences, Toronto, Ontario, Canada $;^{4}$ Institute for Health Policy, Evaluation and Management, University of Toronto, Toronto, Ontario, Canada; ${ }^{5}$ Department of Medical Oncology, Princess Margaret Cancer Centre, Toronto, Ontario, Canada; ${ }^{6}$ Dalla Lana School of Public Health, University of Toronto, Toronto, Ontario, Canada; ${ }^{7}$ Li Ka Shing Knowledge Institute, St. Michael's Hospital, Toronto, 
Ontario, Canada, ${ }^{8}$ Melbourne School of Population and Global Health, University of Melbourne, Melbourne, Victoria, Australia

Abstract Background : Location of cancer care (LOC: pediatric versus adult center) impacts outcomes in adolescents and young adults (AYA) with some cancer types. Data on impact of LOC on survival in AYA with osteogenic sarcoma (OGS) and Ewing sarcoma (EWS) are limited.Objectives : To compare differences in demographics, disease/treatment characteristics, and survival in a population-based cohort of AYA with OGS or EWS treated at pediatric versus adult centers Methods : The IMPACT Cohort captured demographic, disease, and treatment data for all AYA (15-21 years old) diagnosed with OGS and EWS in Ontario, Canada between 1992-2012. Patients were linked to provincial administrative healthcare databases. Outcomes were compared between patients treated in pediatric versus adult centers using appropriate statistical methods.Results : 137 AYA were diagnosed with OGS (LOC: 47 pediatric, 90 adult) and 84 with EWS (LOC: 38 pediatric, 46 adult). AYA treated at pediatric centers were more likely to be enrolled in a clinical trial (OGS 55\% vs 1\%, [p<0.001]; EWS $53 \%$ vs $2 \%$, [p <0.001]) and received higher cumulative chemotherapy doses. Five-year event-free survival (EFS \pm Standard Error) in OGS and EWS were $47 \% \pm 4$ and $43 \% \pm$ 5 , respectively. In multivariable analysis, the impact of LOC (pediatric vs adult center) on EFS in OGS (adjusted hazard ratio [HR] 1.15, 95\% CI 0.58-2.27, $\mathrm{P}=0.69$ ) and EWS (adjusted HR 1.82, 95\% CI 0.97-3.43, $\mathrm{P}=0.06)$ were not statistically significant.Conclusion : Outcomes did not differ by LOC suggesting that AYA with bone tumors can be treated at either pediatric or adult centers.KEYWORDS : adolescent and young adult, Ewing sarcoma, event-free survival, locus of care, osteosarcoma, population-based

\section{1 | INTRODUCTION}

Osteogenic sarcoma (OGS) is the most prevalent primary bone cancer, accounting for almost two-thirds of all bone malignancies and is the most common primary bone cancer in children and adolescents. ${ }^{1,2}$ Ewing sarcoma (EWS) is the second most prevalent bone cancer in all ages. ${ }^{3}$ Both tumors are common and cause considerable morbidity and mortality in the adolescent and young adult population (AYA; ages of 15 to 39 years) ${ }^{4}$

The location of cancer therapy or locus of care (LOC: pediatric versus adult centers) may impact outcomes in AYA with cancer. Variations in care according to LOC, including type and intensity of therapy, access to clinical trials, and treatment team experience may result in differences in survival outcomes. ${ }^{5.6}$

Differences in outcomes according to LOC have been studied in AYA with leukemia, lymphoma, brain tumors, and soft tissue sarcomas. ${ }^{5,7-15}$ However, few studies have compared treatment differences between pediatric and adult centers and explored the impact of LOC on outcomes in bone tumors. ${ }^{12,13,16}$ Differences in treatment intensity and/or time to local therapy are among the possible reasons for outcome disparities in AYA bone tumors. ${ }^{12,13,16}$ The existence of such LOC-based disparities would inform policy and may improve bone tumor outcomes. However, prior studies have been limited by small patient numbers and inconsistent results.

It is a common assumption that prolonged diagnostic interval length (DIL) for cancer has a negative influence on survival because of a risk for more advanced stage disease at diagnosis. ${ }^{17}$ Delays in the diagnosis and initiation of treatment of bone cancers are common, as their symptoms are often vague and misleading or may be misattributed to other pathologies. ${ }^{18}$ However, most prior studies have not shown any association between prolonged DIL and survival in OGS or EWS. ${ }^{19-21}$

We used a population-based provincial cancer registry, to explore outcome disparities among AYA with OGS or EWS treated at pediatric versus adult cancer centers, and to examine the impact of DIL on survival.

\section{2 | METHODS}

\section{1 | Study design, population, and data resources}

After obtaining Research Ethics Board approval from the Hospital for Sick Children and Sunnybrook Health Sciences Centre, we carried out a population-based retrospective cohort study using the IMPACT 
Cohort. The need for individual consent was waived. The creation of the cohort has been described in detal previously. ${ }^{5}$ It comprises all Ontario residents, aged 15 to 21 years at diagnosis of one of six common cancers (including bone tumors) treated at an adult center (a Regional Cancer Center or a community hospital) or a pediatric center (one of the five specialized, tertiary care centers) from 1992 to $2012 .{ }^{5}$ In Ontario, AYA aged less than 18 may receive care at either pediatric or adult centers, whereas older AYA ([?]18) almost always are treated in adult centers. ${ }^{7,9}$ IMPACT was built using two data sources. The Pediatric Oncology Group of Ontario Networked Information System (POGONIS) database captures demographic, disease- and treatment-related data of children diagnosed with cancer before the age of 18 who received their care at a pediatric center. ${ }^{22,23}$ AYA diagnosed between ages 15 to 21 who received their cancer care at an adult center were identified through the Ontario Cancer Registry (OCR) and their clinical data collected by trained chart abstractors. ${ }^{5,7}$ These data were combined to form the IMPACT database. All AYA patients diagnosed with OGS or EWS, except those who lived or were diagnosed out of the province (Ontario), were included in this study. The IMPACT database was linked deterministically using encrypted personal identifiers to population-based health services databases (CIHI-DAD for hospitalization records, NACRS for emergency room visits, OHIP for outpatients visits and physician's billing information; see Appendix 1 ) hosted at ICES (formerly, the Institute for Clinical Evaluative Sciences).

\section{2 | Outcome and variables}

The primary outcome was event-free survival (EFS), defined as the time between primary diagnosis and the occurrence of a first event (progression, relapse, subsequent malignant neoplasm [SMN], or death) or the end of follow-up (December 31, 2018). Most events were captured through chart abstraction. To avoid missing events that occurred at centers different from the primary treatment center, validated health administrative data-based algorithms using billings for chemotherapy, radiation, or palliative care with service dates after the end of the initial therapy were utilized. ${ }^{24}$

The main exposure variable was a 3-level categorical variable combining age and LOC. Pediatric LOC included patients aged 15 to 17.9 years old who were treated at a pediatric center. Adult [?]18 LOCcomprised 18 to 21 years old patients who were treated at an adult center. Patients in pediatric age group ( $<18$ years) who were treated at an adult facility or diagnosed at a pediatric center but treated at an adult center, were categorized as adult $<18 L O C$. A patient's main treating institution was categorized based on delivery of the majority of chemotherapy and/or radiotherapy during first three months after diagnosis. Patients who received upfront chemotherapy at a pediatric center and radiation at an adult center, were considered as pediatric-center patients.

Demographic and patient-related variables included sex and socioeconomic variables (neighborhood median income quintile, rurality, immigrant status). Neighborhood median income quintile was determined utilizing the Canadian census and respective postal code at the date closest to the date of cancer diagnosis. Patients were classified as having urban or rural residence according to their residential postal code at diagnosis and the Rurality Index for Ontario (RIO)-2004. ${ }^{25}$ The immigration status of each patient was determined by utilizing the Citizenship and Immigration Canada (CIC) database and recorded as a binary (yes/no) variable. The main disease-level variables were histology (OGS or EWS), DIL, metastatic status at diagnosis (localized versus metastatic), and site of the primary tumor (limb versus axial).

To calculate DIL, all healthcare claims and visit records in the year preceding cancer diagnosis were organized as discrete episodes and each episode was assigned a single ICD code representing the main purpose (symptom or diagnosis) of that episode. Two pediatric oncologists $(P N$ and $S G$ ) defined the diagnostic codes that were consistent with an underlying OGS or EWS diagnosis, based on their clinical expertise. The DIL for each patient was calculated as the time interval between the date of the first relevant physician encounter with a definite OGS- or EWS-related symptom and the date of cancer diagnosis. To evaluate the association between DIL and survival, categorical variables with two levels according to the calculated median DIL (i.e., short delay [?] median and prolonged delay > median) were created separately for OGS and EWS.

Treatment variables other than LOC included clinical trial enrollment (enrolled vs non-enrolled), time from 
diagnosis to local therapy, and chemotherapy cumulative doses $\left(\mathrm{mg} / \mathrm{m}^{2}\right)$. Time to local therapy was defined as the time from the date of diagnosis to the date of first definitive local treatment (surgery and/or radiation) and was categorized as early ([?] 3 months) versus late ( $>3$ months). The cumulative doses of chemotherapeutic agents were identified and recorded. The Cyclophosphamide Equivalent Dose (CED) was calculated for alkylating agents, using the following equation: ${ }^{26}$

CED $\left(\mathrm{mg} / \mathrm{m}^{2}\right)=1.0$ (cumulative cyclophosphamide dose $\left.\left(\mathrm{mg} / \mathrm{m}^{2}\right)\right)+0.244$ (cumulative

ifosfamide $\operatorname{dose}\left(\mathrm{mg} / \mathrm{m}^{2}\right)$ )

\subsection{Statistical analysis}

Descriptive statistics were generated to describe demographic, disease characteristics, and treatment variables to compare the distributions between patients treated at pediatric versus adult cancer centers, using chisquare tests for categorical variables and t-tests or Kruskal-Wallis tests for continuous variables.

Kaplan-Meier survival analysis was performed to determine EFS. The log-rank test was used to compare survival between groups. Univariate and multivariable Cox proportional hazard $(\mathrm{PH})$ survival analyses were performed to identify factors associated with EFS. Cox PH survival analysis results were presented as hazard ratios (HR) with $95 \%$ confidence intervals (CI). Variables that had a p-value less than 0.1 in univariate analysis were included in multivariable analysis. Prognostic factors previously reported in the literature (LOC, primary site, and sex in OGS and LOC and primary site in EWS) were included a priori in multivariable analysis.

All analyses were performed using SAS (Enterprise Guide) statistical software for Unix environment, version 9.4 (SAS Institute Inc, Cary, NC, USA). Statistical significance was defined as p-value less than 0.05. All data are reported as per the Strengthening the Reporting of Observational Studies in Epidemiology (STROBE) guideline (Appendix 2).

\section{3 | RESULTS}

The cohort included 221 patients (137 OGS, 47 pediatric-center and 90 adult-center and 84 EWS, 38 pediatric-center and 46 adult-center). The demographic, patient, disease, and treatment characteristics of the patients, stratified by locus of care, are shown in Table 1 .

\subsection{Osteogenic sarcoma}

\subsubsection{Demographic, diagnosis, disease, and treatment variables}

As demonstrated in Table $\mathbf{1}$, patients treated at a pediatric center were statistically significantly younger $(15.57+-0.85$ years vs $17.99+-1.50$ years, $\mathrm{p}<0.001)$ and more likely to have metastases than those treated at an adult center $(36.2 \%$ vs $16.7 \%, \mathrm{p}=0.01)$. Clinical trial enrollment was significantly more common in pediatric centers compared to adult centers $(55 \%$ versus $1 \%, \mathrm{p}<0.001)$. The distribution of sex, DIL, socioeconomic variables, and bone primary site did not differ between pediatric and adult centers.

There was no difference in the type of local therapy or time to local therapy in pediatric versus adult centers.

The median cumulative doses of methotrexate, doxorubicin, and the CED were all significantly and substantially higher in pediatric centers than adult centers.

\subsection{2 | Survival outcomes}

The median follow-up time was 8.1 years (Interquartile range [IQR] 2.0 - 17.1 years; range 0.0 -26.7 years). Among patients with localized disease, EFS was the longest in the patients aged 18 or older who received care in adult centers followed by pediatric-center patients and adult-center patients younger than 18 years, respectively (5-year EFS +- SE: $66 \%+-7 \%$ vs $53 \%+-9 \%$ vs $43 \%+-9 \%$; p=0.04) (Fig. 1A and 1B ). Females had significantly better survival outcome than males (5-year EFS +- SE: $76 \%$ +- $7 \%$ vs $46 \%$ +- 6 ; $\mathrm{p}=0.006$ ). Among those with metastatic disease, higher 5-year EFS rates were observed in the patients who received higher doses of doxorubicin $(36 \%+-15 \%$ vs $6 \%+-6 \%$; $=0.004)$, methotrexate $(44 \%+-17 \%$ vs 
$5 \%+-5 \% ; \mathrm{p}=0.001)$, and cisplatin $(31 \%+-13 \%$ vs $7 \%+-6 \% ; \mathrm{p}=0.03)$. None of tumor primary site, income quintile, rurality, immigration status, DIL, clinical trial enrollment, or time to local therapy were associated with EFS in localized or metastatic cohorts.

Table 2 shows the results of univariate and multivariable Cox proportional hazards survival analyses in both localized and metastatic OGS cohorts. Multivariable analysis in the localized cohort revealed only the statistically significant association of sex with EFS; (i.e., males had inferior EFS compared to females). In the metastatic cohort, only the association of higher cumulative doses of methotrexate, doxorubicin and cisplatin with better EFS were significant in multivariable analyses.

\subsection{Ewing sarcoma}

\subsubsection{Demographic, diagnosis, disease, and treatment variables}

As shown in Table 1, patients treated at a pediatric center were more likely to be younger and have limb involvement than those treated at an adult center. Clinical trial enrollment was significantly more frequent in pediatric centers versus adult centers $(53 \%$ versus $2 \%, \mathrm{p}<0.001)$. Sex, DIL, and socioeconomic status did not differ by LOC.

Pediatric centers provided their EWS patients with local therapy (either surgery only or radiation only or both surgery and radiation) earlier than adult centers, regardless of the type of local therapy (Table 3 ). Time to local therapy was not significantly different between localized and metastatic patients. There was no statistically significant difference in radiation doses between pediatric and adult centers.

The most commonly used chemotherapeutic agents were cyclophosphamide, doxorubicin, ifosfamide and etoposide. The median cumulative doses of cyclophosphamide, ifosfamide, etoposide, and the CED were significantly higher in pediatric center patients compared with adult center patients.

\subsection{2 | Survival outcomes}

The median follow-up time was 6.6 years (IQR 1.7 to 14.1 years; range 0.5-25.2 years). None of the variables (including locus of care/age and primary site) was associated with EFS in localized cohort (Fig. 2A and 2B ). In metastatic cohort, only receiving higher dose of etoposide was significantly associated with higher EFS rate (5-year EFS +- SE: $25 \%+-11 \%$ vs 0; 0.0001).

Table 4 demonstrates the results of Cox proportional hazards survival analyses in both localized and metastatic EWS cohorts. In univariate analysis of the localized disease cohort, none of the variables including locus of care/age and primary tumor location showed statistically significant association with survival. In multivariable analysis, none of the variables was significantly associated with EFS (Table 4 ). In the metastatic cohort multivariable analysis, only the association of cumulative doses of etoposide showed statistically significant association with EFS (Table 4 ).

\section{4 | DISCUSSION}

In this retrospective population-based cohort study of AYA with OGS or EWS, we found that despite significant differences in clinical and treatment exposures between patients treated at pediatric versus adult centers (Table 1 ), the location of treatment did not impact survival for either disease.

Previous studies have shown that AYA with ALL or Hodgkin lymphoma have superior survival when treated at pediatric centers. ${ }^{7,10}$ This has been attributed to disparities in treatment regimens and chemotherapy dose intensity, clinical trial enrollment, and treatment team experience ${ }^{11,12,14}$ However, there are few studies that have compared the impact of clinical and treatment differences between pediatric and adult centers on the outcomes in AYA with bone tumors. ${ }^{12,13,16}$

Howell et al. investigated the impact of LOC on survival outcomes in a retrospective study of 1,751 cancer patients (including 76 patients with OGS or EWS) aged 0 to 19 years, treated at one of five US pediatric cancer centers (Children's Oncology Group [COG] members) or in a non-COG ("adult") institution. ${ }^{13}$ Only $36 \%$ of patients, aged 15 to 19 years were treated at a COG (pediatric) institution. In AYA patients with 
"pediatric type" cancers (i.e., cancers that are more common in pediatric age groups: OGS, EWS, Hodgkin lymphoma, ALL, neuroblastoma, rhabdomyosarcoma), those treated at pediatric centers had superior survival outcomes than those who received care at non-pediatric institutions. However, these differences were not statistically significant, possibly due to the small number of the patients. ${ }^{13}$ In a subsequent report, Bleyer et al. re-analyzed the same data to assess the likelihood of treatment of the type of cancer ("pediatric vs adult-type") by oncologists with pediatric versus adult cancer experience. ${ }^{12}$ The results revealed that AYA with "pediatric-type" cancers had better survival at COG (pediatric) centers and those with adult-type tumors (e.g. non-Hodgkin lymphoma) showed a better survival at non-COG (adult) institutions. ${ }^{12}$ The authors concluded that the AYA patients with pediatric types of cancer (including OGS and EWS) fare better if their care is delivered by pediatric oncologists. ${ }^{12}$

In an institution-based retrospective study of 53 patients (29 pediatric and 24 adult) with localized EWS, Gupta et al. studied survival outcomes among those treated at an adult or a pediatric tertiary cancer care center in Ontario between 1990 and 2005. ${ }^{16}$ The median age for the pediatric and adult patients was 13.4 years (range, 0.29-16.2 years) and 26.1 years (range, 16.7-66.5 years), respectively. The total cumulative doses of ifosfamide and cyclophosphamide were lower $(\mathrm{p}<0.0001)$ and the time-to-local therapy was longer (7.4 months vs 3.7 months; $\mathrm{p}=0.0003$ ) in the adult center compared to the pediatric center. The 3 -year EFS rates in pediatric and adult patients were $70 \%$ and $43 \%(\mathrm{p}=0.1)$ and the 3 -year OS rates were $81 \%$ and $59 \%$ $(\mathrm{p}=0.02)$, respectively. The authors concluded that the inferior survival outcome of EWS patients treated at an adult center may be related to lower cumulative doses of alkylating agents and a delay in local therapy in adult-center patients. ${ }^{16}$ In our localized EWS cohort, none of LOC, primary cancer site or time to local therapy was associated with survival.

In our study, the reason for superior EFS in patients with metastatic disease who received higher cumulative doses of methotrexate, doxorubicin, and cisplatin in OGS or cisplatin in EWS is unclear. One possibility is that the higher dose of chemotherapy effectively impacts the survival of patients with metastasis. The second, more likely possibility is that patients with more aggressive disease may have received lower doses of chemotherapeutic agents with a goal of controlling symptoms and avoiding toxicities, or that more aggressive tumors progressed leading to discontinuation of primary therapy, thus confounding the relationship between chemotherapy dose and survival. Unfortunately, the relevant data needed to classify metastatic patients according to their disease severity and aggressiveness were not available to test this hypothesis. The prognostic impact of cumulative doses of chemotherapeutic agents are not separately described in papers that have investigated OGS and EWS prognostic factors. In some studies, the response to neoadjuvant chemotherapy, measured by necrosis extent in surgical specimens, has been considered as a prognostic factor. ${ }^{27,28,29}$ We did not have access to pathology data from excised tumors, so could not evaluate this relationship.

Some studies have shown superior outcomes for patients who treated at pediatric centers with high rate of participation in clinical trials. ${ }^{30-33}$ But some authors argue that patients enrolled in clinical trials might not represent the general population and thus the results might be biased. ${ }^{11}$ In our study, about half of pediatric-center patients with OGS and EWS and only a few adult-center patients were enrolled in clinical trials. Neither EFS nor OS rates significantly differed in patients who were enrolled versus those who were not enrolled in clinical trials.

In our study and several other reports ${ }^{19-21}$, there was no association between DIL and survival in either OGS or EWS. Goedhart et al. reported that prolonged DIL does not necessarily result in inferior survival in OGS and EWS and concluded that the impact of tumor location and its resectability on survival is more than the effect of delay in diagnosis. ${ }^{20}$

The strength of this study, as one of the few studies in literature that compares outcomes of AYA OGS and EWS in pediatric versus adult centers, was the use of health administrative data along with population-based databases. This combination of datasets provided us with an opportunity to capture the relevant health data of the entire provincial population and to assess a wide range of demographic, disease- and treatment-related variables and their impact on outcome as well as with access to possible interactions with the health care system. Using this type of data, improved the generalizability of the results and ensured that our findings 
were not susceptible to recall bias and poor documentation seen in studies based on chart abstraction or questionnaires.

One of the issues in evaluating the prognostic effect of age in OGS, EWS, and other cancers is disparities in age ranges and in definitions of pediatric, adolescent and young adult age groups in various studies. The limited age range of AYA (15 to 21 years) in our study reduced its sample size and hence its power and generalizability and made it difficult to compare our results with those of other studies that included a wider age ranges of AYA patients. Another limitation was missing data in some recognized prognostic factors such as tumor size, response to neoadjuvant chemotherapy (tumor necrosis) and surgical margin status; so that we had to exclude these variables from the study.

In conclusion, survival outcomes in AYA with OGS and EWS in our study did not differ by LOC. This stands in contrast to AYA cancers such as ALL and Hodgkin lymphoma, where studies have shown superior survival associated with treatment at pediatric centers. In this study, even though AYA with bone tumors treated at pediatric centers received higher cumulative doses of chemotherapy than in adult centers, the differences did not impact outcomes. Lack of outcome differences by LOC in our study might be explained by the similarities of treatment team expertise and therapeutic regimens for bone tumors and also biologic characteristics of the patients (given the narrow AYA age range) between pediatric and adult cancer centers. Furthermore, although data was not collected, all bone tumors in Ontario are operated on by specialized orthopedic oncologists; the quality of surgical resection is known to be an important prognostic factor in those with localized bone tumors. ${ }^{34,35}$

Future studies can work on extending the AYA age range to above 21 years by either chart abstraction or linkage to other administrative databases in order to provide a larger sample size and to address the uncertainties about the prognostic impact of LOC, age, cumulative doses of chemotherapeutic agents, and other covariates.

ABBREVIATIONS : AYA, adolescents and young adults; CI, confidence interval; CIC, the Citizenship and Immigration Canada database; COG, Children's Oncology Group; Cox PH survival analysis, Cox proportional hazard survival analysis; EFS, event-free survival; EWS, Ewing sarcoma; HR, hazard ratio; ICES, Institute for Clinical Evaluative Sciences; IMPACT, the Initiative to Maximize Progress in Adolescent and Young Adult Cancer Therapy; IQR, interquartile range; OCR, Ontario Cancer Registry; OGS, osteogenic sarcoma; POGONIS, Pediatric Oncology Group of Ontario Networked Information System; SCC, specialized cancer center; SMN; subsequent malignant neoplasm

\section{CORRESPONDENCE}

Paul C. Nathan, MD, MSc, The Hospital for Sick Children, 555 University Avenue, Toronto, Ontario, Canada M5G 1X8. Email: paul.nathan@sickkids.ca

\section{CONFLICT OF INTEREST}

The authors declare that there is no conflict of interest.

\section{ACKNOWLEDGMENTS AND DISCLOSURES}

This study is funded by a Canadian Institutes for Health Research Team Grant. Parts of the material used in this study are based on data and information provided by the Canadian Institute for Health Research (CIHR) and ICES. ICES is supported in part by a grant from the Ontario Ministry of Health and Long-Term Care. The ideas, results, and conclusions expressed herein are those of the authors and do not necessarily reflect the opinions of the Ministry of Health and Long-Term Care, ICES, or CIHR.

\section{DATA AVAILABILITY STATEMENT}

The data that support the finding of this study utilized the health administrative data, which contains personal health information. Privacy legislation in Ontario, Canada prevents these data from being made publicly available. 


\section{REFERENCES}

1) Ferguson JL, Turner SP. Bone cancer: diagnosis and treatment principles. Am Fam Physician 2018; 98(4):205-213.

2) Zhang Y, Yang J, Zhao N, et al. Progress in the chemotherapeutic treatment of osteosarcoma (Review). Oncology Letters 2018;16:6228-6237.

3) Zhang L, Zhou X, Liu X, et al. Advances in multi-therapies for the treatment of Ewing's sarcoma. Transl Surg 2017;2:14-8. 4) Fukushima T, Ogura K, Akiyama T, et al. Descriptive epidemiology and outcomes of bone sarcomas in adolescent and young adult patients in Japan. BMC Musculoskeletal Disorders 2018;19:297. 5) Baxter NN, Daly C, Gupta S, et al. The Initiative to Maximize Progress in Adolescent and Young Adult Cancer Therapy (IMPACT) Cohort Study: a population-based cohort of young Canadians with cancer. BMC Cancer 2014;14:805. 6) De P, Ellison LF, Barr RD, et al. Canadian adolescents and young adults with cancer: opportunity to improve coordination and level of care. CMAJ 2011; 183(3): E194-197. 7) Gupta S, Pole JD, Baxter NN, et al. The effect of adopting pediatric protocols in adolescents and young adults with acute lymphoblastic leukemia in pediatric vs adult centers: An IMPACT Cohort study. Cancer Med 2019;8:20952103. 8) Gupta S, Baxter NN, Pole J, et al. Outcome Among Adolescents and Young Adults with Acute Myeloid Leukemia at Pediatric Versus Adult Centers: A Population-Based Study Using the IMPACT Cohort. Blood 2019;134 (Supplement-1): 4749. 9) Gupta S, Baxter NN, Hodgson D, et al. Treatment patterns and outcomes in adolescence and young adults with Hodgkin lymphoma in pediatric versus adult centers: An IMPACT Cohort Study. Cancer Med 2020;9:6933- 6945. 10) Henderson TO, Parsons SK, Wroblewski KE. Outcomes in Adolescents and Young Adults With Hodgkin Lymphoma Treated on US Cooperative Group Protocols: An Adult Intergroup (E2496) and Children's Oncology Group (COG AHOD0031) Comparative Analysis. Cancer 2018;124:136-44. 11) Pole JD, Alibhai SMH, Ethier MC, et al. Adolescents with acute lymphoblastic leukemia treated at pediatric versus adult hospitals. Annals of Oncology 2013;24: 801- 806. 12) Bleyer A. The quid pro quo of pediatric versus adult services for older adolescent cancer patients. Pediatr Blood Cancer 2010;54:238-241. 13) Howell DL, Ward KC, Austin HD, et al. Access to Pediatric Cancer Care by Age, Race, and Diagnosis, and Outcomes of Cancer Treatment in Pediatric and Adolescent Patients in the State of Georgia. J Clin Oncol 2007; 25:4610-4615. 14) Mitchell AE, Scarcella DL, Rigutto GL, et al. Cancer in adolescents and young adults: treatment and outcome in Victoria. MJA 2004;180:59-62. 15) Furlong W, Rae C, Greenberg ML, et al. Surveillance and survival among adolescents and young adults with cancer in Ontario, Canada. Int. J. Cancer 2012;131:2660-2667. 16) Gupta AA, Pappo A, Saunders $\mathrm{N}$, et al. Clinical outcome of children and adults with localized Ewing sarcoma. Impact of chemotherapy dose and timing of local therapy. Cancer 2010;116 (13): 3189-94. 17) Lethaby CD, Picton S, Kinsey SE, et al. A systematic review of time to diagnosis in children and young adults with cancer. Arch Dis Child 2013;98:349-355. 18) Schnurr C, Pippan M, Steutzer H, et al. Treatment delay of bone tumors, compilation of a sociodemographic risk profile: A retrospective study. BMC Cancer 2008; 8: 22. 19) Goyal S, Roscoe J, Ryder WDJ, et al. Symptom interval in young people with bone cancer. European Journal of Cancer 2004; 40: 2280-2286. 20) Goedhart LM, Gerbers JG, Ploegmaker JJW, et al. Delay in diagnosis and its effect on clinical outcome in high-grade sarcoma of bone: a referral oncological study. Orthopaedic Surgery 2016;8:122-128. 21) Brasme J, Chalumeau M, Oberlin O, et al. Time to diagnosis of Ewing tumors in children and adolescents is not associated with metastasis and survival: A prospective multicenter study of 436 patients. J Clin Oncol 2014; 32: 1935-1940. 22) Agha M, DiMonte B, Greenberg M. Incidence trends and projections for childhood cancer in Ontario. Int J Cancer 2006;118:2809-2815. 23) Greenberg ML, Barr RD, Dimonte B, et al. Childhood cancer registries in Ontario, Canada: lessons learned from a comparison of two registries. Int J Cancer 2003;105:88-91. 24) Gupta S, Nathan PC, Baxter NN, et al. Validity of administrative data in identifying cancer-related events in adolescents and young adults: A populationbased study using the IMPACT Cohort. Med Care 2018; 56: e32-e38. 25) Kralj B. "Measuring 'rurality' for purposes of health-care planning: an empirical measure for Ontario". Ontario Medical Review. October 2000. 26) Green DM, Nolan VG, Goodman PJ, et al. The Cyclophosphamide Equivalent Dose as an approach for quantifying alkylating agent exposure. A report from the Childhood Cancer Survivor Study. Pediatr Blood Cancer 2014; 61(1): 53-67. 27) Saeter G, Elomaa I, Wahlqvist Y, et al. Prognostic factors in bone 
sarcomas. Acta Orthopaedica Scandinavica 1997; 68:sup273, 156-160, DOI:10.1080/17453674.1997.11744723. 28) Petrilli AS, Gentil FC, Epelman S, et al. Increased survival, limb preservation, and prognostic factors for osteosarcoma. Cancer 1991; 68:733-737. 29) Bielack SS, Kempf-Bielack B, Delling G, et al. Prognostic factors in high-grade osteosarcoma of the extremities or trunk: An analysis of 1,702 patients treated on Neoadjuvant Cooperative Osteosarcoma Study Group protocols. J Clin Oncol 2002; 20:776-790. 30) Boissel N, Auclerc MF, Lheritier V, et al. Should adolescents with acute lymphoblastic leukemia be treated as old children or young adults? Comparison of the French FRALLE-93 and LALA-94 trials. J Clin Oncol 2003, 21 (5): 774-780. 31) Epelman S. The Adolescent and Young Adult with Cancer: State of the Art-Brain Tumor. Curr Oncol Rep 2013;15:308-316. 32) De Bont JM, Holt Bvd, Dekker AW et al.. Significant difference in outcome for adolescents with acute lymphoblastic leukemia treated on pediatric vs adult protocols in The Netherlands. Leukemia 2004; 18: 2032-2035. 33) Ramanujachar R, Richards S, Hann I et al. Adolescents with acute lymphoblastic leukaemia: outcome on UK National paediatric (ALL97) and adult (UKALLXII/ E2993) trials. Pediatric Blood Cancer 2007; 48: 254-261. 34) Basile P, Greengard E, Weigel B, Spector L. Prognostic factors for development of subsequent metastases in localized osteosarcoma: A systematic review and identification of literature gaps. Sarcoma 2020 Mar 18;2020:7431549. doi:10.1155/2020/7431549. eCollection 2020. 35) Stachelek GC, Ligon JA, Vogel J, et al. Predictors of recurrence and patterns of initial failure in localized Ewing sarcoma: A contemporary 20-Year Experience. Sarcoma, volume 2021 Article ID 6681741 | https://doi.org/10.1155/2021/6681741

\section{LEGENDS}

FIGURE 1 Event-free survival of AYA with osteogenic sarcoma by locus of care: A) Localized B) Metastatic FIGURE 2 Event-free survival of AYA with Ewing sarcoma by locus of care: A) Localized B) Metastatic

TABLE 1 Demographic, patient, disease, diagnosis, and treatment characteristics, stratified by locus of care

TABLE 2 Factors associated with event-free survival in adolescents and young adults with osteogenic sarcoma TABLE 3 Ewing sarcoma, time to definitive local therapy (Days)

TABLE 4 Factors associated with event-free survival in adolescents and young adults with Ewing sarcoma

\section{Hosted file}

Table 1.docx available at https://authorea.com/users/436266/articles/538711-impact-of-locusof-care-on-outcomes-in-adolescents-and-young-adults-with-osteogenic-sarcoma-and-ewingsarcoma-treated-at-pediatric-versus-adult-cancer-centers-an-impact-cohort-study

\section{Hosted file}

Table 2.docx available at https://authorea.com/users/436266/articles/538711-impact-of-locusof-care-on-outcomes-in-adolescents-and-young-adults-with-osteogenic-sarcoma-and-ewingsarcoma-treated-at-pediatric-versus-adult-cancer-centers-an-impact-cohort-study

\section{Hosted file}

Table 3.docx available at https://authorea.com/users/436266/articles/538711-impact-of-locusof-care-on-outcomes-in-adolescents-and-young-adults-with-osteogenic-sarcoma-and-ewingsarcoma-treated-at-pediatric-versus-adult-cancer-centers-an-impact-cohort-study

\section{Hosted file}

Table 4.docx available at https://authorea.com/users/436266/articles/538711-impact-of-locusof-care-on-outcomes-in-adolescents-and-young-adults-with-osteogenic-sarcoma-and-ewingsarcoma-treated-at-pediatric-versus-adult-cancer-centers-an-impact-cohort-study

\section{Hosted file}


Figure 1.pdf available at https://authorea.com/users/436266/articles/538711-impact-of-locusof-care-on-outcomes-in-adolescents-and-young-adults-with-osteogenic-sarcoma-and-ewingsarcoma-treated-at-pediatric-versus-adult-cancer-centers-an-impact-cohort-study

\section{Hosted file}

Figure 2.pdf available at https://authorea.com/users/436266/articles/538711-impact-of-locusof-care-on-outcomes-in-adolescents-and-young-adults-with-osteogenic-sarcoma-and-ewingsarcoma-treated-at-pediatric-versus-adult-cancer-centers-an-impact-cohort-study 